\title{
The Antimicrobial Peptides Psoriasin (S100A7) and Koebnerisin (S100A15) Suppress Extracellular Matrix Production and Proliferation of Human Fibroblasts
}

\author{
Gerd G. Gauglitz Daniela Bureik Stephanie Zwicker Thomas Ruzicka Ronald Wolf \\ Department of Dermatology and Allergy, Ludwig-Maximilian University, Munich, Germany
}

\section{Key Words}

Fibroblasts · Psoriasin (S100A7) · Koebnerisin (S100A15) ·

Wound healing $\cdot$ Excessive scarring

\begin{abstract}
Background/Aims: Keloids result from aberrations in the normal wound healing cascade and can lead to pruritus, contractures and pain. The underlying mechanisms of excessive scarring are not yet understood, and most therapeutic strategies remain unsatisfactory. Psoriasin (S100A7) and koebnerisin (S100A15) are released by keratinocytes during physiological wound healing. We found $\mathrm{S} 100$ production is markedly decreased in keloid scar tissue. The disturbed epidermal S100 expression might contribute to keloid formation; thus, we studied their effect on dermal fibroblasts and extracellular matrix (ECM) production. Methods: S100 peptides, ECM regulation and distribution were analysed in normal and keloid tissue by quantitative PCR (qPCR), immunoblotting and immunofluorescent staining. Isolated dermal fibroblasts were incubated with $\mathrm{S} 100$ proteins, and the regulation of ECM and transforming growth factor (TGF)- $\beta$ was determined using qPCR. Fibroblast proliferation and viability were determined by the 5-bromo-2'-deoxyuridine assay and crystal violet assay. Results: Keloid tissue featured a pronounced expression of ECMs, such as collagen types 1
\end{abstract}

(c) 2014 S. Karger AG, Basel

$1660-5527 / 14 / 0283-0115 \$ 39.50 / 0$ and 3, whereas the production of psoriasin and koebnerisin was markedly decreased in keloid-derived cells and keloid tissue. Both $\mathrm{S} 100$ proteins inhibited the expression of collagens, fibronectin- 1 , $\alpha$-smooth-muscle actin and TGF- $\beta$ by fibroblasts. Further, they also suppressed fibroblast proliferation. Conclusion: Psoriasin and koebnerisin show antifibrotic effects and may lead to novel preventive and therapeutic strategies for fibroproliferative diseases.

두 2014 S. Karger AG, Base

\section{Introduction}

Excess scar formation occurs after dermal injury as a result of abnormal wound healing. Both hypertrophic scars and keloids are associated with significant morbidity by causing pruritus, pain and/or contractures, which significantly affect the patient's quality of life [1]. Despite the relatively high prevalence of keloids and hypertrophic scars in the general population, the molecular mechanisms underlying excessive scar formation are not fully understood. Most therapeutic strategies remain unsatisfactory due to poor knowledge of the complex mechanisms underlying the process of excessive scarring [2-4].

Excessive scar tissue formation results from increased fibroblast proliferation and disturbed extracellular ma-

\section{KARGER 125}

E-Mail karger@karger.com www.karger.com/spp
Gerd G. Gauglitz, MD, MMS

Department of Dermatology and Allergy

Ludwig-Maximilian University

DE-Munich (Germany)

E-Mail Gerd.Gauglitz@med.uni-muenchen.de 
trix (ECM) deposition in the dermis. Keloids and hypertrophic scars are densely populated by inflammatory cells that release fibrogenic factors, such as transforming growth factor- $\beta$ (TGF- $\beta$ ). This environment leads to increased transcription and translation of collagen I and III, fibronectin and laminin, as well as deficient ECM degradation and remodelling [5]. Recent evidence suggests that both the severity of inflammation and the type of immune response predispose to excessive scar formation [5]. Development of a Th2 response promotes fibrogenesis, whereas a Th1 predominance attenuates tissue fibrosis [6, 7]. Current research in keloid pathophysiology further suggests that epidermal-dermal interactions [8] and alterations of the fibroblast phenotype through several (growth) factors and downstream signalling pathways [9] may be involved in excessive scarring.

Recently, high levels of psoriasin (S100A7) proteins have been detected in human wound exudate and granulation tissue [10]. Immunohistological studies suggest that psoriasin is produced by keratinocytes surrounding the wound and is released into the wound exudate to inhibit bacterial survival [11]. S100 proteins are effectors of calcium-dependent processes and intervene with the control of cell cycle, cell growth and cell differentiation as well as chemoattractants for inflammatory cells $[12,13]$. Hence, the expression of $\mathrm{S} 100$ proteins has been associated with epidermal maturation, inflammation and wound healing [14-16].

Koebnerisin (S100A15) protein shares 93\% overall identity with psoriasin, with differences mainly concerning the deduced $\mathrm{N}$-terminal $\mathrm{Ca}^{2+}$-binding site [17]. Koebnerisin expression is induced in cultured human keratinocytes upon treatment with tumour necrosis factor- $\alpha$ and interferon (IFN)- $\gamma$, and interleukin- $1 \beta$, suggesting that the pro-inflammatory environment in diseased skin contributes to koebnerisin expression in the epidermis. A similar regulation pattern through Th1 cytokines has been shown for psoriasin with coregulation of both psoriasin and koebnerisin in inflammation [18]. In fact, compared with normal skin, both psoriasin and koebnerisin proteins are upregulated in inflamed lesional skin, such as in psoriasis or chronic atopic eczema [18]. Since we observed that patients suffering from psoriasis rarely develop keloids upon dermal injury (observation of the authors), we hypothesized that psoriasin/koebnerisin proteins may have antifibrotic effects. Indeed, here, we could demonstrate that epidermal psoriasin and koebnerisin alone or in combination have suppressive effects on ECM production and fibroblast proliferation in vitro.

\section{Materials and Methods}

\section{Patients and Skin Samples}

Sample acquisition was approved by the local ethical committee (Faculty of Medicine, Ludwig-Maximilian University, Munich, Germany). The study was conducted according to the Declaration of Helsinki principles. For all procedures, informed written consent was obtained from the patients. Skin biopsies were taken from patients $(n=9)$ who underwent surgical removal of keloid tissue. Gender- and age-matched tissue specimens from patients $(n=9)$ with no history of excessive scarring served as normal controls.

\section{Isolation and Cell Culture of Human Fibroblasts}

Primary fibroblasts were isolated from normal skin. Subcutaneous fat was removed from the respective tissue specimens and the remaining tissue was washed in phosphate-buffered saline. In order to isolate the dermis, specimens were incubated overnight at $4^{\circ} \mathrm{C}$ in sterile Dispase II (Roche) solution $(4 \mathrm{mg} / \mathrm{ml})$. The isolated dermis was minced into small pieces, and explants placed on culture dishes were overlaid with Dulbecco's modified Eagle's medium that was supplemented with $10 \%$ fetal bovine serum and penicillin $(100 \mathrm{U} / \mathrm{ml})$, and streptomycin $(100 \mu \mathrm{g} / \mathrm{ml}$; PAA Laboratories, Pasching, Austria), and incubated at $37^{\circ} \mathrm{C}$ and $5 \% \mathrm{CO}_{2}$. Skin-derived fibroblasts were grown in culture for 3-4 weeks and then passaged after trypsinization. Early passages of fibroblasts were seeded at a density of $3 \times 10^{4}$ cells into 12 -well plates and switched to serumfree medium at $70-80 \%$ confluence $24 \mathrm{~h}$ prior to exposure to IFN- $\gamma$ (100 ng/ml; Biomol, Hamburg, Germany), psoriasin (S100A7) and koebnerisin (S100A15; 0.01-1 $\mu \mathrm{g} / \mathrm{ml}$ ) alone or in combination.

\section{RNA Isolation and Quantitative Real-Time PCR}

Cultured cells were harvested, and RNA was isolated using the RNA Miniprep Kit (Zymo Research, Irvine, Calif., USA). cDNA synthesis was performed with $1 \mu \mathrm{g}$ of total RNA using the DyNAmo cDNA Synthesis Kit (Finnzymes, Espo, Finland). Expression of COL1A1, COL1A2 und COL3A1, TGF- $\beta_{1},-\beta_{2},-\beta_{3}$, fibronectin-1, laminin- $\beta_{2}$ and $\alpha$-smooth-muscle actin were normalized against $\beta$-actin using commercial gene-specific primers (Qiagen, Hilden, Germany). Quantitative PCR (qPCR) assays were run in the CFX96 Real Time System as specified by the manufacturer (BioRad, Hercules, Calif., USA). All analyses were performed in triplicate from 2 to 3 independent cell stimulation experiments.

\section{Cell Viability and Proliferation Assays}

Fibroblast viability was determined by crystal violet assay. Cells were exposed to koebnerisin (S100A15) and psoriasin (S100A7) alone or in combination for $24 \mathrm{~h}$ and then stained with crystal violet ( 0.5 in $20 \%$ methanol) for $20 \mathrm{~min}$ at room temperature. Then, cells were decolorized with $0.1 \mathrm{M}$ sodium citrate in $50 \%$ ethanol, and absorbance was read at $550 \mathrm{~nm}$ using a microplate reader (Spectra MR, Dynex Technology, Chantilly, Va., USA).

Fibroblast proliferation was determined by the 5-bromo-2'-deoxyuridine (BrdU) Assay (Roche Applied Science, Mannheim, Germany) according to the manufacturer's instructions. Cells were exposed to IFN- $\gamma(100 \mathrm{ng} / \mathrm{ml})$, koebnerisin (S100A15) and psoriasin (S100A7) alone or in combination for $48 \mathrm{~h}$. At $24 \mathrm{~h}, \mathrm{BrdU}$ labelling medium was added, and incoporated BrdU during cell proliferation was detected by an anti-BrdU horseradish peroxidase-labelled antibody at $370 \mathrm{~nm}$ using a microplate reader (Spectra MR, Dynex Technology). 
Immunofluorescence Staining and Immunoblotting

Immunofluorescence staining was performed on serial 5- $\mu \mathrm{m}$ frozen sections of human normal and keloid skin and on primary fibroblasts derived from normal skin and keloids. Plated cells and skin sections were fixed in acetone and blocked in 10\% normal goat serum. After overnight incubation with anti-S100A15 $(5 \mu \mathrm{g} / \mathrm{ml}$, customized), anti-S100A7 ( $1 \mu \mathrm{g} / \mathrm{ml}$, Abcam, Cambridge, UK) and anti-vimentin (1:50, Sigma Aldrich, Steinheim, Germany) antibody, sections and cells were then incubated with an Alexa Fluor 647-labelled goat anti-rabbit antibody (Invitrogen, Carlsbad, Calif., USA) and with a tetramethylrhodamine-conjugated goat antimouse antibody (Dianova, Hamburg, Germany) diluted in $10 \%$ normal goat serum and incubated for $1 \mathrm{~h}$ at room temperature in a dark humidified chamber. Staining with secondary antibodies only was performed as a control for unspecific antibody binding. Sections were overlaid with 4',6-diamidino-2-phenylindole (ProLong Gold antifade reagent, Invitrogen). Images of fluorescent stained tissues were recorded using a 12-bit CCD digital camera PCO PixelFly (PCO, Kelheim, Germany) on a fluorescent microscope Zeiss Imager Z1 (Zeiss, Jena, Germany). Immunoblotting was performed as previously described [19]. In brief, skin or fibroblast lysates ( $15 \mu \mathrm{g} / \mathrm{lane}$ ) were prepared using radioimmunoprecipitation assay buffer. Proteins were separated using a $12 \%$ sodium dodecyl sulphate polyacrylamide gel, transferred to polyvinylidene fluoride membranes, blocked and incubated with anti-hS100A15 (5 $\mu \mathrm{g} / \mathrm{ml}$, Costum), anti-S100A7 antibody $(1 \mu \mathrm{g} /$ $\mathrm{ml}, \mathrm{Abcam})$ and anti- $\beta$-actin $(1 \mu \mathrm{g} / \mathrm{ml}$, Cell Signaling, Danvers, Mass., USA) overnight. After incubation for $1 \mathrm{~h}$ with horseradish peroxidase-conjugated secondary antibodies (anti-rabbit, Cell Signaling; anti-mouse, Pierce), proteins were detected by chemoluminescence.

\section{Statistical Analysis}

All statistical analyses were performed using GraphPad Prism 4.0 (GraphPad Software Inc.). Student's t test was used to calculate statistical differences. Values of $\mathrm{p}<0.05$ were considered significant, and all data are displayed as means \pm SD.

\section{Results}

Psoriasin (S100A7) and Koebnerisin (S100A15) Are

Decreased in Keloid Tissue and Differently Produced by Normal and Keloid Fibroblasts

Our data showed that psoriasin (S100A7) and koebnerisin (S100A15) are expressed in the upper differentiated epidermal layers of normal skin (fig. 1A, a, c). Compared to psoriasin, koebnerisin was also detectable at the epidermal-dermal junction. In keloid tissue sections, staining of either $\mathrm{S} 100$ protein was reduced within the epidermis or underlying dermis (fig. 1A, b, d). We further identified normal fibroblasts as an additional cellular source for koebnerisin (fig. 1B, a, c). Koebnerisin was distributed throughout the cellular cytoplasm and partially colocalized with the mesenchymal marker vimentin. Keloid-derived fibroblasts showed a similar expression pat- tern, but stained more weakly for koebnerisin (fig. 1B, b, d), while psoriasin was not detectable in both normal and keloid-derived fibroblasts (fig. 1B, e, f). Immunoblot analysis in whole tissue showed psoriasin- and koebnerisinreactive bands in normal skin (fig. 1C). The psoriasin and koebnerisin protein expression varied among the individual skin tissue samples, but was overall weaker in keloid tissue when normalized to $\beta$-actin (fig. 1D). Thus, the coregulation of psoriasin and koebnerisin and their distribution in different cellular compartments in the skin suggest their functional contribution in keloid tissues.

\section{Psoriasin (S100A7) and Koebnerisin (S100A15) Are}

Associated with Reduced Collagen Expression in the

Skin and Inhibit Collagen Expression by Fibroblasts

Keloids form after prolonged inflammation that leads to an increased proliferation and activation of fibroblasts with enhanced deposition of disorganized collagen fibres of types 1 and 3 in the dermis [20]. Whole tissue analysis demonstrated that COL1A1, COL1A2 and COL3A1 were produced in normal skin (fig. 2A). Skin biopsies from keloid tissue showed an increased production of the investigated collagen types, which participate in fibrotic scarring. In corresponding samples, both psoriasin (S100A7) and koebnerisin (S100A15) alternate transcripts (S100A15L and S100A15S) were present in normal skin. However, both $S 100$ peptides were downregulated in the keloid tissue and revealed a reverse $S 100$-collagen regulation pattern under fibrotic conditions (fig. 2B, compare fig. 1). Data implied that psoriasin and koebnerisin affect collagen production in fibroblasts. Cultured fibroblasts were then exposed to increasing $\mathrm{S} 100$ peptide concentrations, and their effect on collagen expression was measured. Data showed that recombinant psoriasin decreased expression of COL1A1, COL1A2 and COL3A1 at various concentrations and significantly reduced levels of COL3A1 compared to controls (fig. 2C). Also, recombinant koebnerisin significantly decreased expression of COL1A1 and COL3A1 at most of the concentrations utilized (fig. 2D). Exposure of cultured fibroblasts to psoria$\sin$ and koebnerisin at effective collagen-suppressing concentration did not influence the number of viable cells as determined by the crystal violet assay (data not shown).

\section{Psoriasin (S100A7) and Koebnerisin (S100A15)}

Synergize to Attenuate Expression of ECM Proteins by Fibroblasts

Psoriasin (S100A7) and koebnerisin (S100A15) are coregulated under pathological conditions and show synergistic effects important for disease pathogenesis [21, 22]. As 


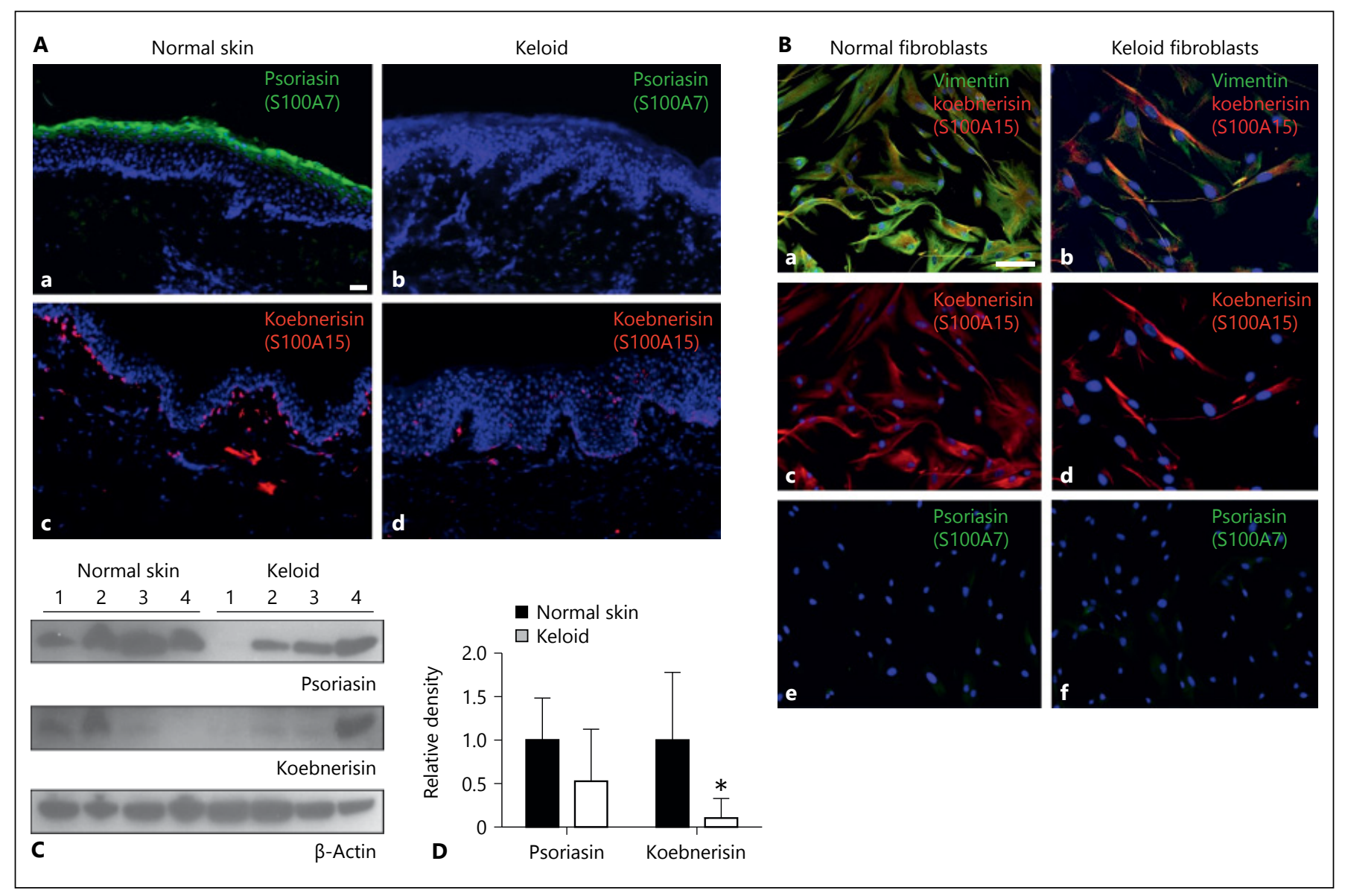

Fig. 1. Psoriasin (S100A7) and koebnerisin (S100A15) expression and distribution in keloid tissue and keloid fibroblasts. A Frozen skin tissue sections from normal subjects $(\mathbf{a}, \mathbf{c})$ and patients with keloids (b, d) immunostained for psoriasin (S100A7) and koebnerisin (S100A15). Scale bar $=100 \mu \mathrm{m}$. B Cultured fibroblasts isolated from normal skin $(\mathbf{a}, \mathbf{c}, \mathbf{e})$ and keloid biopsies $(\mathbf{b}, \mathbf{c}, \mathbf{f}) \mathrm{immu}$ -

both peptides individually regulate collagen expression in fibroblasts (fig. 2C, D), their synergistic effect on fibroblast activity was investigated. When used in combination, the suppressing effect of psoriasin and koebnerisin on fibroblast activity was further enhanced for all investigated collagen types (COL1A1, COL1A2, COL3A1; fig. 3A). Next, cultured fibroblasts were exposed to psoriasin and koebnerisin peptides at concentrations that were effective to suppress the regulation of collagens. Data showed that each recombinant psoriasin and koebnerisin peptide decreased the expression of fibronectin in fibroblasts (fig. 3B). Their suppressing effect was further enhanced when both peptides were used in combination and was more effective than IFN- $\gamma$ (positive control, fig. 3B). The combination of psoriasin and koebnerisin also significantly inhibited the ex- nostained for vimentin, psoriasin and koebnerisin. Scale bar $=100$ $\mu \mathrm{m}$. C Total protein lysates of normal skin and keloid scar tissue (15 $\mu \mathrm{g} / \mathrm{lane})$ were probed with specific antibodies against psoriasin and koebnerisin. D Psoriasin and koebnerisin staining intensities were normalized against $\beta$-actin. ${ }^{*} \mathrm{p}<0.05$ determined by Student's $t$ test.

pression of laminin- $\beta_{2}$ and $\alpha$-smooth-muscle actin in fibroblasts (fig. 3C, D). Cultured fibroblasts exposed to either recombinant psoriasin or koebnerisin peptides slightly affected the expression of TGF- $\beta_{1},-\beta_{2}$ and $-\beta_{3}$, where koebnerisin had the most pronounced effect on TGF- $\beta_{3}$ (fig. 3E). When used in combination, psoriasin and koebnerisin significantly reduced expression of all investigated TGF- $\beta$ isoforms. Compared to the S100 peptides, IFN- $\gamma$ suppressed the expression of TGF- $\beta_{2}$ and $-\beta_{3}$ in cultured fibroblasts, but did not regulate TGF- $\beta_{1}$ expression.

\section{Psoriasin (S100A7) and Koebnerisin (S100A15)}

Synergize to Suppress Fibroblast Proliferation

To gain insight into other antifibrotic effects of the $\mathrm{S} 100$ peptides, we investigated the functional importance of 


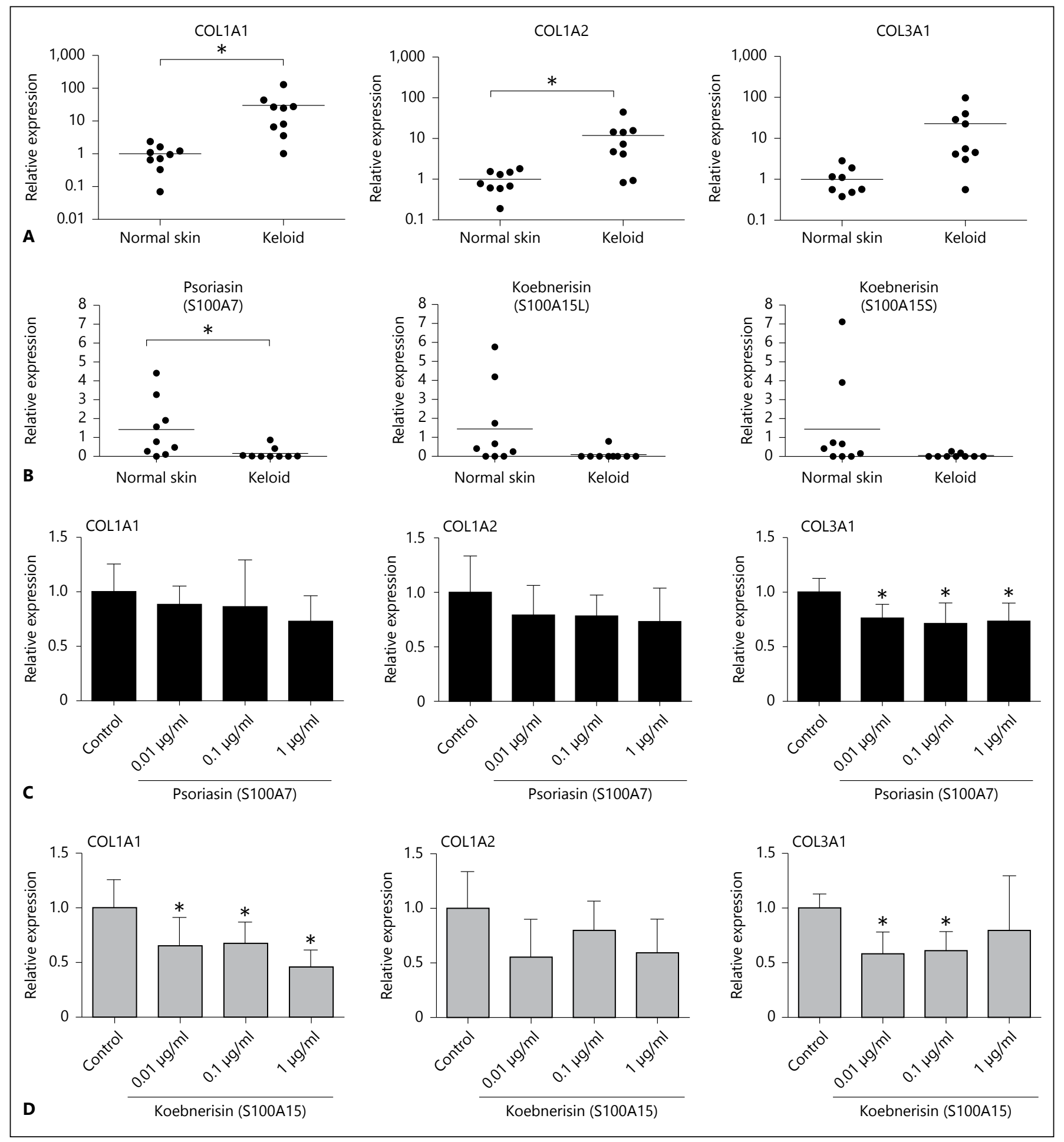

Fig. 2. Psoriasin (S100A7) and koebnerisin (S100A15) mark decreased collagen expression in the skin and inhibit collagen expression by fibroblasts. Total RNA was extracted from biopsies of normal skin $(n=9)$ and keloid scar tissue $(n=9)$, and real-time qPCR was performed to assay collagen types 1 and 3 (COL1A1, COL1A2, COL3A1; A) and psoriasin (S100A7) and koebnerisin (S100A15-S,
S100A15-L) transcripts (B) using gene-specific primers. Cultured fibroblasts were treated with indicated concentrations of psoriasin (S100A7; C) and koebnerisin (S100A15; D), and collagen type 1 and 3 expression was analysed by real-time qPCR. $*$ p $<0.05$ determined by Student's t test. 


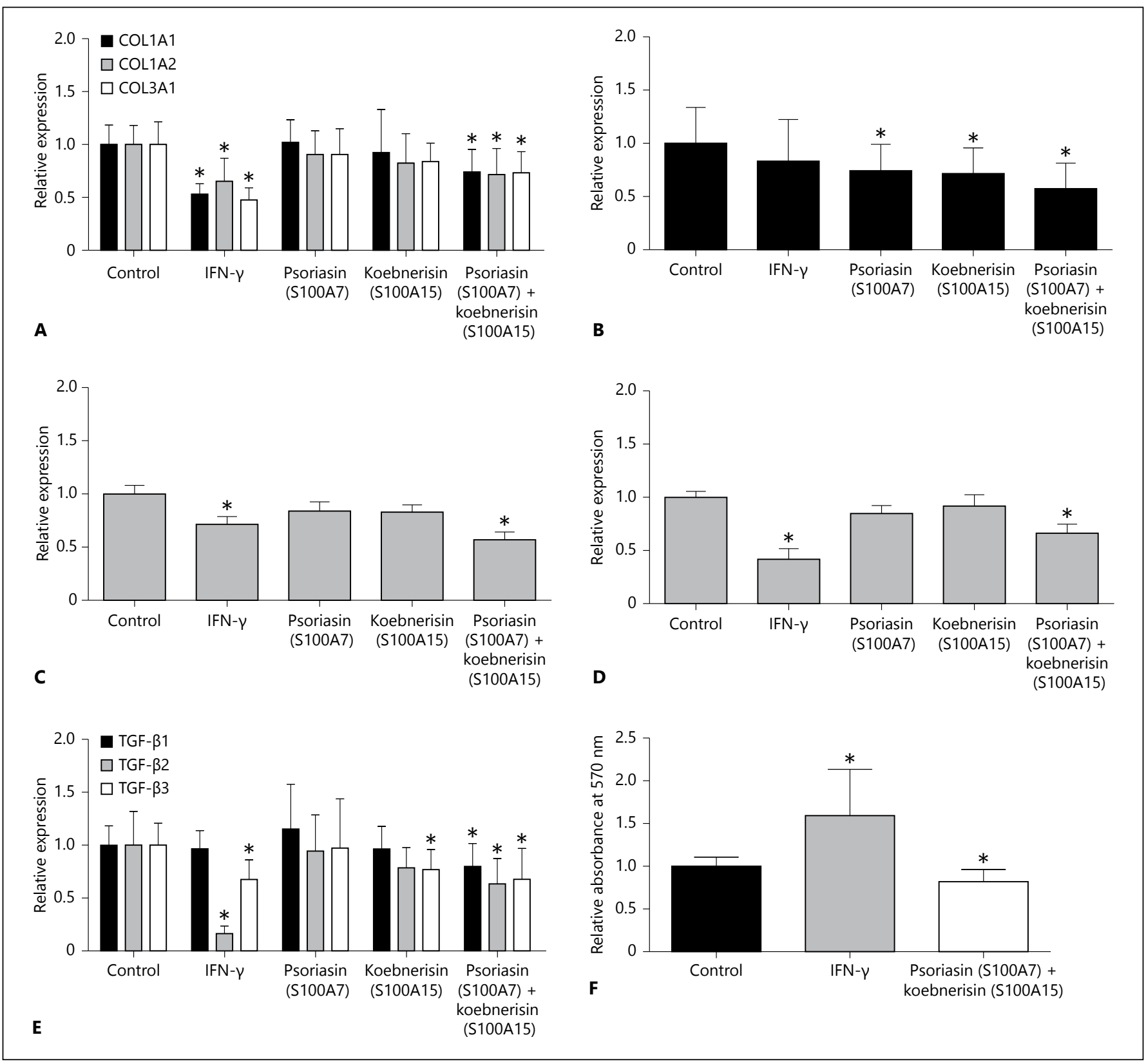

Fig. 3. Psoriasin (S100A7) and koebnerisin (S100A15) synergize to suppress ECM expression by fibroblasts and inhibit fibroblast proliferation. A-F Cultured fibroblasts were treated with IFN- $\gamma$ $(0.1 \mu \mathrm{g} / \mathrm{ml})$, psoriasin (S100A7) and koebnerisin (S100A15) alone or in combination. Total RNA was extracted, and real-time qPCR

psoriasin (S100A7) and koebnerisin (S100A15) on fibroblast proliferation. Cultured fibroblasts were exposed to psoriasin and koebnerisin peptides at concentrations that were effective to suppress the expression of ECM components. Each recombinant psoriasin and koebnerisin peptide significantly inhibited the proliferation of fibroblasts

was performed to assay collagen types 1 and 3 (COL1A1, COL1A2, COL3A1; A), fibronectin-1 (B), laminin- $\beta_{1}(\mathbf{C})$, $\alpha$-smooth-muscle actin (D) and TGF- $\beta$ types 1-3 (E) using gene-specific primers. F Proliferation of cultured fibroblasts was analysed by BrdU incorporation after treatment with IFN- $\gamma$, psoriasin and koebnerisin.

as measured by reduced BrdU uptake (data not shown). The antiproliferative effect was further enhanced when both S100 peptides were used in combination (fig. 3F). In contrast, incubation of fibroblasts with IFN- $\gamma$ increased BrdU uptake, which is indicative of a stimulation of fibroblast proliferation. 


\section{Discussion}

Transformation of a wound clot into granulation tissue requires a delicate balance between ECM protein deposition and degradation, and when disrupted, abnormalities in scarring appear [1]. Despite a plethora of in vivo and in vitro studies investigating the complex mechanisms underlying hypertrophic scar formation, its pathophysiology remains poorly understood. Diverse proteins have been suggested to be involved in excessive scar formation; however, to date, little attention has been paid to the $S 100$ proteins.

Genes of the S100 family encode small (9-13 kDa), calcium-binding proteins of the EF hand type that regulate tissue maturation, inflammation and antimicrobial defence [12]. Dysregulated levels of certain S100 peptides have previously been detected during wound repair of epithelia and other tissues [14-16,23]. Upregulated in the hyperproliferative epidermis, S100A8 and S100A9 promote the growth of epithelial keratinocytes [24-26], whereas S100A2 drives TGF- $\beta$-dependent mesenchymal cell migration [20]. S100B mediates myofibroblast proliferation and might contribute to the scar formation observed in the infarcted myocardium [27]. Increased fibroblast activation and chronic fibrotic kidney disease have been associated with $\mathrm{S} 100 \mathrm{~A} 4$, which has been established as a diagnostic marker for systemic fibrotic disorders [28].

In this context, we aimed to elucidate the significance of two S100 proteins, psoriasin (S100A7) and koebnerisin (S100A15), for the pathogenesis of keloid formation.

Psoriasin and koebnerisin form a unique subgroup of the $\mathrm{S} 100$ protein family and were discovered to be upregulated in psoriatic skin [17, 29]. Although being highly homologous peptides, psoriasin and koebnerisin have distinct functions as epidermal antimicrobial peptides, leucocyte attractants and pro-inflammatory 'alarmins' [18]. Compared with psoriasin, koebnerisin is also expressed by additional cell types in the skin, such as endothelial cells and smooth muscle cells. Here, we noticed a predominent interface pattern (fig. 1A), which may also be due to the fact that staining intensity varies among the different cell types and depends on the imaging conditions. We also discovered that skin fibroblasts serve as another source of koebnerisin (fig. 1B). Interestingly, when compared to normal skin, staining of keloid tissue sections for both S100 proteins was significantly reduced within the epidermis and underlying dermis.

S100 Proteins Suppress Fibroblast Activity
Both koebnerisin mRNA isoforms lead to the same protein and were expressed in normal fibroblasts, but were downregulated in keloid-derived fibroblasts. Psoriasin transcript, however, could not be detected in either type of fibroblasts. Whole tissue immunoblot analysis further demonstrated that psoriasin and koebnerisin staining intensity was overall weaker in keloid tissue when compared with normal skin (fig. 1).

Recent evidence suggests that the type of immune response predisposes to excessive scarring [5]. While the development of a Th2 response has been strongly linked to fibrogenesis, a predominance of Th1 CD41 cells has been shown to almost completely attenuate the formation of tissue fibrosis via production of IFN- $\gamma[6,7]$. Both psoriasin and koebnerisin are inducible by Th1-derived cytokines in epidermal keratinocytes $[11,30]$, and the lack of inducibility of both S100 by Th2-derived cytokines may account for the low expression levels of psoriasin and koebnerisin in keloid tissue [31].

Importantly, low $\mathrm{S} 100$ protein expression corresponded to high levels of profibrotic markers, such as collagens, in keloid tissue (fig. 2A, B). This data suggests that subepidermal fibroblasts in keloid tissue might be exposed to decreased levels of secreted psoriasin and koebnerisin that, in turn, could affect the production of collagens and other extracellular matrix compounds.

In fact, when fibroblasts were exposed to either $S 100$ peptide, the expression of both collagen types 1 and 3 was attenuated (fig. 2C, D; fig. 3A). Also, both psoriasin and koebnerisin peptides individually decreased the expression of other ECM compounds, such as fibronectin-1, laminin- $\beta_{2}$ and $\alpha$-smooth-muscle actin, without affecting fibroblast viability. The suppressing patterns of psoriasin and koebnerisin on the ECM components were different and are in line with distinct functional mechanisms of these S100 peptides. Despite their high homology, extracellular psoriasin and koebnerisin activate different classes of receptors [21]. Psoriasin activates the pattern recognition receptor 'receptor of advanced glycated end products', whereas koebnerisin functions through a pertussis toxin-sensitive Gi protein-coupled receptor. When coregulated under pathophysiological conditions, both S100 peptides can exert synergistic effects. Here, we could show that co-exposure of fibroblasts to psoriasin and koebnerisin leads to a synergistic inhibition of the ECM components (fig. 3). In some instances, their combined effect was comparable to that of IFN- $\gamma$, which has previously been established as one of the most potent inhibitors of ECM-related genes, such as type I collagen, fibronectin and laminin [32-34]. 
TGF- $\beta$ signalling has been implicated as key regulator in early stages of ECM production and in keloid formation. While TGF- $\beta_{1}$ and $-\beta_{2}$ represent the most important stimulators of collagen and proteoglycan synthesis $[35,36]$, TGF- $\beta_{3}$ reduces connective tissue deposition [37]. We could demonstrate that the combination of psoriasin and koebnerisin significantly attenuated the expression of TGF- $\beta$ subtypes 1-3; however, the suppressive effects of IFN- $\gamma$ on TGF- $\beta_{2}$ and $-\beta_{3}$ were much more pronounced. Nevertheless, incubation of cultured fibroblasts with psoriasin and koebnerisin did significantly attenuate fibroblast proliferation, which could account for the suppressive effects of psoriasin (S100A7) and koebnerisin (S100A15) on
ECM production. Thus, although the exact molecular mechanism underlying the S100 antifibrotic effects remains currently unclear, a better understanding of these two proteins may give additional insights into the complex pathophysiology of fibroproliferative diseases of the skin.

\section{Acknowledgements}

The authors thank Michael Muehlstaedt and Christian Kunte for their excellent technical assistance and tissue sample collection. This work was supported by grants of the Ludwig-Maximilian University Munich (FoeFoLe, No. 640) and the German Research Foundation (DFG, Wo 843/3-1).

\section{References}

1 Gauglitz GG, Korting HC, Pavicic T, Ruzicka T, Jeschke MG: Hypertrophic scarring and keloids: pathomechanisms and current and emerging treatment strategies. Mol Med 2011;17:113-125.

$\checkmark 2$ De Paepe K, Sieg A, Le Meur M, Rogiers V: Silicones as nonocclusive topical agents. Skin Pharmacol Physiol 2014;27:164-171.

3 Gauglitz GG: Management of keloids and hypertrophic scars: current and emerging options. Clin Cosmet Investig Dermatol 2013;6: 103-114.

4 Gauglitz GG, Bureik D, Dombrowski Y, Pavicic T, Ruzicka T, et al: Botulinum toxin A for the treatment of keloids. Skin Pharmacol Physiol 2012;25:313-318.

5 Brown JJ, Bayat A: Genetic susceptibility to raised dermal scarring. Br J Dermatol 2009; 161:8-18.

6 Wynn TA: Fibrotic disease and the $\mathrm{T}(\mathrm{H}) 1 /$ $\mathrm{T}(\mathrm{H}) 2$ paradigm. Nat Rev Immunol 2004;4: 583-594.

$>7$ Doucet C, Brouty-Boye D, Pottin-Clemenceau C, Canonica GW, Jasmin C, et al: Interleukin (IL) 4 and IL-13 act on human lung fibroblasts. Implication in asthma. J Clin Invest 1998;101:2129-2139.

8 Armour A, Scott PG, Tredget EE: Cellular and molecular pathology of HTS: basis for treatment. Wound Repair Regen 2007;15(suppl 1): S6-S17.

-9 Butler PD, Longaker MT, Yang GP: Current progress in keloid research and treatment. J Am Coll Surg 2008;206:731-741.

10 Lee KC, Eckert RL: S100A7 (psoriasin) mechanism of antibacterial action in wounds. J Invest Dermatol 2007;127:945-957.

11 Gläser R, Harder J, Lange H, Bartels J, Christophers E, et al: Antimicrobial psoriasin (S100A7) protects human skin from Escherichia coli infection. Nat Immunol 2005;6:5764.

12 Berridge MJ, Bootman MD, Roderick HL: Calcium signalling: dynamics, homeostasis and remodelling. Nat Rev Mol Cell Biol 2003; 21 Wolf R, Howard OM, Dong HF, Voscopoulos 4:517-529.

13 Donato R: S100: a multigenic family of calcium-modulated proteins of the EF-hand type with intracellular and extracellular functional roles. Int J Biochem Cell Biol 2001;33:637668.

14 Kim EJ, Helfman DM: Characterization of the metastasis-associated protein, S100A4 Roles of calcium binding and dimerization in cellular localization and interaction with myosin. J Biol Chem 2003;278:3006330073.

15 Meltzer JC, Sanders V, Grimm PC, Stern E, Rivier C, et al: Production of digoxigenin-labelled RNA probes and the detection of cytokine mRNA in rat spleen and brain by in situ hybridization. Brain Res Brain Res Protoc 1998;2:339-351.

16 Schafer BW, Heizmann CW: The S100 family of EF-hand calcium-binding proteins: functions and pathology. Trends Biochem Sci 1996;21:134-140.

17 Wolf R, Mirmohammadsadegh A, Walz M, Lysa B, Tartler U, et al: Molecular cloning and characterization of alternatively spliced mRNA isoforms from psoriatic skin encoding a novel member of the S100 family. FASEB J 2003;17:1969-1971.

18 Wolf R, Ruzicka T, Yuspa SH: Novel S100A7 (psoriasin)/S100A15 (koebnerisin) subfamily: highly homologous but distinct in regulation and function. Amino Acids 2011;41:789796.

19 Wolf R, Mascia F, Dharamsi A, Howard $\mathrm{OM}$, Cataisson C, et al: Gene from a psoriasis susceptibility locus primes the skin for inflammation. Sci Transl Med 2010;2: 61 ra90.

20 Naz S, Ranganathan P, Bodapati P, Shastry $\mathrm{AH}$, Mishra LN, et al: Regulation of S100A2 expression by TGF-beta-induced MEK/ERK signalling and its role in cell migration/invasion. Biochem J 2012;447:81-91.
C, Boeshans K, et al: Chemotactic activity of S100A7 (psoriasin) is mediated by the receptor for advanced glycation end products and potentiates inflammation with highly homologous but functionally distinct S100A15. J Immunol 2008;181:1499-1506.

22 Hegyi Z, Zwicker S, Bureik D, Peric M, Koglin $S$, et al: Vitamin D analog calcipotriol suppresses the Th17 cytokine-induced proinflammatory S100 'alarmins' psoriasin (S100A7) and koebnerisin (S100A15) in psoriasis. J Invest Dermatol 2012;132:1416-1424.

23 Ong CT, Khoo YT, Mukhopadhyay A, Masilamani J, Do DV, et al: Comparative proteomic analysis between normal skin and keloid scar. Br J Dermatol 2010;162:13021315 .

24 Benoit S, Toksoy A, Ahlmann M, Schmidt M, Sunderkotter C, et al: Elevated serum levels of calcium-binding $\mathrm{S} 100$ proteins $\mathrm{A} 8$ and $\mathrm{A} 9$ reflect disease activity and abnormal differentiation of keratinocytes in psoriasis. Br J Dermatol 2006;155:62-66.

25 Zenz R, Eferl R, Kenner L, Florin L, Hummerich $\mathrm{L}$, et al: Psoriasis-like skin disease and arthritis caused by inducible epidermal deletion of Jun proteins. Nature 2005;437:369375 .

26 Nukui T, Ehama R, Sakaguchi M, Sonegawa H, Katagiri C, et al: S100A8/A9, a key mediator for positive feedback growth stimulation of normal human keratinocytes. J Cell Biochem 2008;104:453-464. Parker TG: S100B-RAGE dependent VEGF secretion by cardiac myocytes induces myofibroblast proliferation. J Mol Cell Cardiol 2012;52:464-473.

28 Schneider M, Hansen JL, Sheikh SP: S100A4: a common mediator of epithelial-mesenchymal transition, fibrosis and regeneration in diseases? J Mol Med (Berl) 2008;86: 507-522.
27 Tsoporis JN, Izhar S, Proteau G, Slaughter G, 
29 Madsen P, Rasmussen HH, Leffers H, Honore $\mathrm{B}$, Dejgaard K, et al: Molecular cloning, occurrence, and expression of a novel partially secreted protein 'psoriasin' that is highly upregulated in psoriatic skin. J Invest Dermatol 1991;97:701-712.

30 Gläser R, Meyer-Hoffert U, Harder J, Cordes $\mathrm{J}$, Wittersheim $\mathrm{M}$, et al: The antimicrobial protein psoriasin (S100A7) is upregulated in atopic dermatitis and after experimental skin barrier disruption. J Invest Dermatol 2009; 129:641-649.
31 Grewe M, Bruijnzeel-Koomen CA, Schopf E, Thepen T, Langeveld-Wildschut AG, et al: A role for Th1 and Th2 cells in the immunopathogenesis of atopic dermatitis. Immunol Today 1998;19:359-361.

32 Granstein RD, Rook A, Flotte TJ, Haas A, Gallo RL, et al: A controlled trial of intralesional recombinant interferon-gamma in the treatment of keloidal scarring. Clinical and histologic findings. Arch Dermatol 1990;126: 1295-1302.

33 Granstein RD, Flotte TJ, Amento EP: Interferons and collagen production. J Invest Dermatol 1990;95:75S-80S.

-34 Woeckel VJ, Eijken M, van de Peppel J, Chiba $\mathrm{H}$, van der Eerden $\mathrm{BC}$, et al: IFNbeta impairs extracellular matrix formation leading to inhibition of mineralization by effects in the early stage of human osteoblast differentiation. J Cell Physiol 2012;227:2668-2676.
35 Szulgit G, Rudolph R, Wandel A, Tenenhaus $\mathrm{M}$, Panos R, et al: Alterations in fibroblast alphalbetal integrin collagen receptor expression in keloids and hypertrophic scars. J Invest Dermatol 2002;118:409-415.

36 Kose O, Waseem A: Keloids and hypertrophic scars: are they two different sides of the same coin? Dermatol Surg 2008;34:336-346.

37 Bock O, Yu H, Zitron S, Bayat A, Ferguson MW, et al: Studies of transforming growth factors beta 1-3 and their receptors I and II in fibroblasts of keloids and hypertrophic scars. Acta Derm Venereol 2005;85:216-220. 